\title{
Terahertz Spectroscopy for Accurate Identification of Panax quinquefolium Basing on Nonconjugated 24(R)-Pseudoginsenoside $F_{11}$
}

\author{
Tianyi Kou, ${ }^{1}$ Ji Ye, ${ }^{2}$ Jing Wang, ${ }^{3}$ Yan Peng $\mathbb{D}^{1,4}$ Zefang Wang, ${ }^{1}$ Chenjun Shi, ${ }^{1}$ Xu Wu, ${ }^{1,4}$ \\ Xitian Hu, ${ }^{1}$ Haihong Chen, ${ }^{1}$ Ling Zhang, ${ }^{1}$ Xiaohong Chen, ${ }^{1}$ Yiming Zhu ${ }^{\mathbb{D}},{ }^{1,4}$ Huiliang Li $\left(\mathbb{D},{ }^{2}\right.$ \\ and Songlin Zhuang ${ }^{1,4}$ \\ ${ }^{1}$ Terahertz Technology Innovation Research Institute, Shanghai Key Lab of Modern Optical System, Terahertz Science Cooperative \\ Innovation Center, University of Shanghai for Science and Technology, Shanghai Institute of Intelligent Science and Technology, \\ Shanghai, China \\ ${ }^{2}$ Department of Pharmacy, Second Military Medical University, Shanghai, China \\ ${ }^{3}$ School of Pharmacy, Nanjing Medical University, Nanjing, China \\ ${ }^{4}$ Shanghai Institute of Intelligent Science and Technology, Tongji University Shanghai, China
}

Correspondence should be addressed to Yan Peng; py@usst.edu.cn, Yiming Zhu; ymzhu@usst.edu.cn, and Huiliang Li; faranli@hotmail.com

Received 6 July 2020; Accepted 29 October 2020; Published 27 January 2021

Copyright (C) 2021 Tianyi Kou et al. Exclusive Licensee Nanjing Agricultural University. Distributed under a Creative Commons Attribution License (CC BY 4.0).

\begin{abstract}
Panax quinquefolium is a perennial herbaceous plant that contains many beneficial ginsenosides with diverse pharmacological effects. 24(R)-pseudoginsenoside $\mathrm{F}_{11}$ is specific to P. quinquefolium, a useful biomarker for distinguishing this species from other related plants. However, because of its nonconjugated property and the complexity of existing detection methods, this biomarker cannot be used as the identification standard. We herein present a stable 24(R)-pseudoginsenoside $F_{11}$ fingerprint spectrum in the terahertz band, thereby proving that $\mathrm{F}_{11}$ can be detected and quantitatively analyzed via terahertz spectroscopy. We also analyzed the sample by high-performance liquid chromatography-triple quadrupole mass spectrometry. The difference between the normalized data for the two analytical methods was less than 5\%. Furthermore, P. quinquefolium from different areas and other substances can be clearly distinguished based on these terahertz spectra with a standard principal component analysis. Our method is a fast, simple, and cost-effective approach for identifying and quantitatively analyzing P. quinquefolium.
\end{abstract}

\section{Introduction}

Panax quinquefolium, which is a herbaceous species in the family Araliaceae [1], is a valuable medicinal plant in many countries because of its antihypertensive, nerve cell stimulatory, antidiabetic, and anticancer properties. The medicinal qualities of $P$. quinquefolium are due to its diverse ginsenosides that are beneficial to humans, with extensive pharmacological effects on the central nervous system $[2,3]$, cardiovascular system $[4,5]$, endocrine system $[6,7]$, and immune system [8]. Moreover, these compounds are useful for treating cancer [9-11]. To date, more than 60 ginsenosides have been identified in P. quinquefolium [12]. According to the available information in the US and Chinese pharmacopeias, $\mathrm{Rg}_{1}, \mathrm{Rb}_{1}$, Re, and 24(R)-pseudoginsenoside $\mathrm{F}_{11}$ are the most important ginsenosides in P. quinquefolium. Similarly, $\mathrm{Rg}_{1}, \mathrm{Rb}_{1}$, and $\mathrm{Re}$ are the ginsenosides with the most important functions in ginseng. Thus, $\mathrm{F}_{11}$ is the main difference between $P$. quinquefolium and ginseng. Although $P$. quinquefolium and ginseng can be distinguished by traditional methods (e.g., analysis of appearance, application of microscopy, and examination of physical and chemical properties), high-performance liquid chromatography- (HPLC-) based mass spectrometry (MS) now represents the most authoritative and comprehensive method for differentiating between these species. For example, Wang et al. used HPLC-tandem MS (HPLC-MS/MS) and Chan et al. used HPLC-electrospray ionization MS (HPLC-ESI-MS) to 
distinguish $P$. quinquefolium from ginseng based on the distribution of ginsenosides [13, 14]. Ma et al. used HPLC-atmosphere pressure chemical ionization MS (HPLCAPCI-MS) to study the $\mathrm{F}_{11}$ content in P. quinquefolium and revealed that a majonoside isomer and the ginsenoside $\mathrm{Rd}$ may be used as markers to identify $P$. quinquefolium from China and North America [15]. However, these HPLCbased methods are currently useful only for laboratory research because they require complex pretreatment processes and they are costly and time-consuming [16]. Consequently, there is an urgent need for the development of a new, accurate, rapid, and qualitative and quantitative analytical method for identifying $P$. quinquefolium and the subsequent control of any derived drugs.

Researchers have confirmed that $\mathrm{F}_{11}$ is exclusive to $P$. quinquefolium, a unique biomarker for this species [17]. However, $\mathrm{F}_{11}$ is a nonconjugated substance that cannot be detected by a conventional HPLC-ultraviolet MS (HPLCUV-MS) method. Moreover, most liquid chromatography methods cannot distinguish between $\mathrm{F}_{11}$ and the ginsenoside $\mathrm{Rf}$ because of their similar retention times $[13,14]$. Thus, there is currently no available method for the rapid and accurate identification of $\mathrm{F}_{11}$.

The terahertz $(\mathrm{THz})$ wave $(0.1-10 \mathrm{THz})$ lying between the millimeter wave and the infrared band is of significant importance to the biological sciences because of complementary information to traditional spectroscopic measurements on low-frequency bond vibrations, hydrogen bond stretching, and bond torsions in liquids and gases [18]. Therefore, the collective behavior (vibration and rotation) characteristics of biomolecules make $\mathrm{THz}$ spectroscopy a promising sensing modality for clinical diagnosis [19]. Combining with the nondestructive, accurate, rapid, and good penetrability [20], $\mathrm{THz}$ spectroscopy also has many other potential applications in several research fields, including physics [21, 22], biology [23, 24], chemistry [25], and medicine [26].

In this study, we use $\mathrm{THz}$ spectroscopy to analyze $\mathrm{F}_{11}$ and $P$. quinquefolium and do the comparison with other methods. The detailed research contents are as follows: (I) calculate the theoretical vibration modes of $\mathrm{F}_{11}$ in the $\mathrm{THz}$ band; (II) experimentally confirm its characteristic $\mathrm{THz}$ absorption spectrum; (III) examine the THz absorption spectra of different $P$. quinquefolium samples; (IV) compare the quantitative data resulting from $\mathrm{THz}$ spectroscopic and HPLC-triple quadrupole-MS (HPLC-QQQ-MS) methods; and (V) distinguish P. quinquefolium from many similar substances based on the $\mathrm{THz}$ spectral characteristics of $\mathrm{F}_{11}$ via a principal component analysis (PCA). We herein describe a new method for the rapid qualitative and quantitative analysis of $P$. quinquefolium, which is expected to be extended to various plant detection.

\section{Materials and Methods}

2.1. Experimental Materials. The 24(R)-pseudoginsenoside $\mathrm{F}_{11}$ (>98\%, CAS: 69884-00-0) and ginsenoside $\operatorname{Re}(>98 \%$, CAS: 52286-59-6) used in this study were purchased from PureChem Standard (Chengdu, China). Polyethylene (PE) powder (particle size: 40-50 $\mu \mathrm{m}$, CAS: 9002-88-4) was purchased from Sigma-Aldrich (Shanghai, China). Additionally, 14 different batches of $P$. quinquefolium produced in North America and Jilin, China, were used in the form of block roots. All samples were used without further purification.

2.2. Sample Preparation for Terahertz Spectroscopy. Regarding the $\mathrm{THz}$ spectroscopy analysis, the sample preparation required grinding, sieving, and tablet pressing. Specifically, $P$. quinquefolium samples were ground to a powder with the MM400 ball mill (Retsch, Germany) at a vibration frequency of $90 \mathrm{~Hz}$ for $3 \mathrm{~min}$. The powdered samples (particle size: $40-50 \mu \mathrm{m}$ ) were placed under an infrared lamp to keep the sample dry, sieved, and mixed with PE powder $(25 \% \mathrm{w} /$ $w)$ in an agate vessel. Samples were then compressed into tablets ( $1 \mathrm{~mm}$ thick and $13 \mathrm{~mm}$ diameter) with a hydraulic press (4 tons of pressure).

2.3. Sample Preparation for the HPLC Analysis. The P. quinquefolium crude powder $(2.5 \mathrm{~g})$ was added to a $100 \mathrm{~mL}$ round bottom flask, after which $25 \mathrm{~mL}$ methanol (analytical grade, 99.99\%, CAS: 67-56-1; Fisher, USA) was added and the mixture was incubated overnight. The sample was heated under reflux in a $72^{\circ} \mathrm{C}$ water bath for $2 \mathrm{~h}$, cooled, and filtered. The temperature was set according to the boiling point of methanol and the laboratory environment. Next, $25 \mathrm{~mL}$ methanol was added to the residue, after which the sample was heated under reflux for another $2 \mathrm{~h}$, cooled, and then filtered with a qualitative filter paper (Hangzhou, China). The filtrate was added to a $50 \mathrm{~mL}$ volumetric flask, diluted with an equal volume of methanol, and thoroughly mixed before storing at $-20^{\circ} \mathrm{C}[27,28]$.

2.4. Sample Preparation for Additional Analyses. For UV spectroscopy, a $5 \mathrm{mg}$ sample was dissolved in $1 \mathrm{~mL}$ water (analytical grade). The sample was diluted to $0.05 \mathrm{mg} \mathrm{mL}^{-1}$ prior to the analysis.

For Raman spectroscopy, the sample powder produced with the MM400 ball mill was placed on glass slides and analyzed with a Raman spectrometer.

For mid-infrared spectroscopy, the milled sample powder and $\mathrm{KBr}$ (Sigma-Aldrich, CAS: 7758-02-3) were mixed and compressed into tablets (1 mm thick and $13 \mathrm{~mm}$ diameter) with a hydraulic press (4 tons of pressure).

2.5. Experimental Instruments. The Terahertz experimental device is Brooke's Fourier transform infrared spectrometer (vectex80v, Bruker Optics). The far-infrared (IR) light source is a self-cooled mercury lamp, and the detector is a DLATG$\mathrm{S} /$ polyethylene detector. Therefore, the effective coverage of the spectral region is $30-680 \mathrm{~cm}^{-1}$, and the SNR is better than $10000: 1$. In the spectral range of 1.5 to $4 \mathrm{THz}$, with the resolution of $2 \mathrm{~cm}^{-1}$, the scanning times of 128 , and the scanning speed of $5 \mathrm{kHz}$, all the spectra are measured at room temperature $\left(22^{\circ} \mathrm{C}\right)$ in a vacuum environment to reduce the influence of water vapor on the experiment.

The instrument used for HPLC results was Agilent 1200 high-performance liquid chromatography (MA, USA) and 6410 triple quadrupole mass spectrometer (MA, USA). The reagents used are acetonitrile and formic acid 
(chromatographic pure, Fisher, USA; content 88\%, CAS: 64-18-6); methanol (chromatographic pure, Fisher, USA; content 99.99\%, CAS: 67-56-1); ultrapure water (Milli-Q50 SP pure water system); and other reagents (analytic pure).

UV-VIS spectra were recorded using a UV-2450 spectrophotometer (Shimadzu, Japan). The test wavelength range is $190 \mathrm{~nm}-900 \mathrm{~nm}$. Wavelength repetition accuracy is $\pm 0.1 \mathrm{~nm}$.

The Raman spectrometer model is DR-3168-LDC-DD (University of Shanghai for Science and Technology), The microscope in the machine is from Nikon (Japan). The spectral region is $400-3000 \mathrm{~cm}^{-1}$.

The MIR experimental device is Brooke's Fourier transform infrared spectrometer (vectex $80 \mathrm{v}$, Bruker Optics). The effective coverage of the spectral region is $400-7000 \mathrm{~cm}^{-1}$. In the spectral range of 400 to $4000 \mathrm{~cm}^{-1}$, we have the following: the resolution of $2 \mathrm{~cm}^{-1}$, the scanning times of 128 , and the scanning speed of $5 \mathrm{kHz}$.

2.6. Absorption Peak Area Calculation. For the characteristic peak to be calculated, the slope of each point in the corresponding frequency range is calculated. When the slope of a point begins to be bigger than 0.3 , it is regarded as the starting point of the region; when the slope of a point begins to be less than -0.3, it is regarded as the end point of the region. After determining the region range, the integral function of Origin software is used to do the area calculation.

2.7. Sample Preparation for Additional Analyses. The PCA refers to a statistical extraction method for simplifying datasets. It involves a linear transformation, with all principal components uncorrelated and ordered. Each principal component is a linear combination of the original variables. This transformation converts the data to a new coordinate system, with the largest variance on the first coordinate (i.e., first principal component $\left.\left(\mathrm{PC}_{1}\right)\right)$, the second-largest variance on the second coordinate $\left(\mathrm{PC}_{2}\right)$, and so on $\left(\mathrm{PC}_{p}\right)$ (Supplementary material 1 ) [29]. These PCs contribute the most to the variance in the dataset (e.g., material composition and spectral amplitude). Here, the PCA program we used is the function [COEFF SCORE latent $]=\operatorname{princomp}(\mathrm{x})$ built in MATLAB.

\section{Results and Discussion}

3.1. Theoretical Model. 24(R)-pseudoginsenoside $\mathrm{F}_{11}$, with a molecular formula of $\mathrm{C}_{42} \mathrm{H}_{72} \mathrm{O}_{14}$ and a molecular weight of 801.02 [30], is an ocotillol-type saponin (Figure 1(a)) [31, 32]. It is a dammarane-type triterpenoid that contains a fourtrans-ring rigid steroid structure [33-35].

The molecular formula model (from the ChemSpider website, URL: http://www.chemspider.com) was imported into the GaussView quantum chemistry program [36], which applied the B3LYP hybrid functional with the 6-311G basis set. Additionally, the DFT-D dispersion correction was introduced.

3.2. Terahertz Spectroscopy Absorption Spectra. The molecular formula and simulation results for $\mathrm{F}_{11}$ (Figure 1(b)) revealed four characteristic absorption peaks at 1.76, 2.31, 3.15 , and $3.68 \mathrm{THz}$. Analyses of the vibration/rotation of atoms/functional groups in the molecule indicated that the absorption peaks at 1.76 and $3.68 \mathrm{THz}$ are mainly due to the wagging of the $\mathrm{CH}_{3}$ group, whereas the absorption peak at $2.31 \mathrm{THz}$ is mainly the result of the wagging of the $\mathrm{OH}$ group in the hexatomic ring. The absorption peak at $3.15 \mathrm{THz}$ represents the wagging of the $\mathrm{CH}_{2}-\mathrm{OH}$ group (Supplementary material 2).

We also used some traditional detection and comparison methods, including UV, mid-infrared, and Raman spectroscopic analyses. The nonconjugated $\mathrm{F}_{11}$ was compared with the ginsenoside Re, which is a conjugated biomarker of $P$. quinquefolium. Figure 1 (c) presents the UV spectra of $\mathrm{F}_{11}$ and $\operatorname{Re}\left(0.05 \mathrm{mg} \mathrm{mL}^{-1}\right)$. Because the $\pi-\pi *$ transition wavelength range of nonconjugated systems is not within the stable operating range of the UV detector $(190-700 \mathrm{~nm})$, the presence of conjugated systems is determined based on the transition mode of organic compounds. In this study, Re was clearly identified as a molecule containing a conjugated system because of its obvious UV absorption peak at $220 \mathrm{~nm}$. In contrast, a UV absorption peak was not detected for the nonconjugated $F_{11}$. Thus, the UV spectrum was only able to determine the existence of a nonconjugated system but could not reveal its specific properties. The Raman spectra of $F_{11}$ and $\operatorname{Re}$ (Figure 1(d)) included three peaks at 2120, 2435 , and $2943 \mathrm{~cm}^{-1}$, corresponding to the polarization band of $\mathrm{C} \equiv \mathrm{C}-\mathrm{C} \equiv \mathrm{C}$ symmetric stretching vibration and the antisymmetric stretching of $\mathrm{CH}_{2}$, with the same frequency and amplitude [37]. Raman spectroscopy is often used to examine functional groups. The presence of these three functional groups in $\mathrm{F}_{11}$ and other ginsenosides prevents them from being used to differentiate between ginsenoside compounds. In the mid-infrared spectra for $\mathrm{F}_{11}$ and Re (Figure 1(e)), peaks were detected at $1062 \mathrm{~cm}^{-1}(\mathrm{C}-\mathrm{OH}$ stretching vibration), $1382 \mathrm{~cm}^{-1}\left(\mathrm{CH}_{3}\right.$ symmetrical twisting vibration), $1458 \mathrm{~cm}^{-1}\left(\mathrm{CH}_{3}\right.$ asymmetrical twisting vibration), $1648 \mathrm{~cm}^{-1}$ (aromatic C-C stretching vibration), $2927 \mathrm{~cm}^{-1}\left(\mathrm{CH}_{2}\right.$ antisymmetric stretching vibration), and $3400 \mathrm{~cm}^{-1}$ (OH stretching vibration). Similar to the Raman spectroscopy results, the presence of the same peaks in the $\mathrm{F}_{11}$ and Re spectra indicated that the mid-infrared spectroscopy cannot be used for identifying ginsenosides. On the other side, the $\mathrm{THz}$ spectra of $F_{11}$ and $\operatorname{Re}$ (Figure 1(f)) contained clear absorption peaks at $1.76,2.31,3.11$, and $3.61 \mathrm{THz}$ for $\mathrm{F}_{11}$ with a very small error bar, which are consistent with the theoretical data. In contrast, Re lacked an obvious absorption peak between 1.5 and $4.0 \mathrm{THz}$. The difference between Figure 1(e) and (f) is because the $\mathrm{THz}$ spectrum and mid-infrared spectrum have different frequency ranges, which correspond to different vibrational and rotational frequency characteristics of molecules. Here, the $\mathrm{THz}$ spectral fingerprint (low frequency) is more fit for the identification of the $\mathrm{F}_{11}$ and Re.

3.3. Comparison of Different Producing Areas. Because the growth of medicinal herbs is closely related to the natural environment (e.g., soil, water quality, and climate), their quality and efficacy vary among growing regions [38, 39]. Therefore, herbs from different locations need to be clearly distinguished and then used to produce specific medicines. In this study, we selected seven $P$. quinquefolium batches from North America (Canada and USA) and another seven 


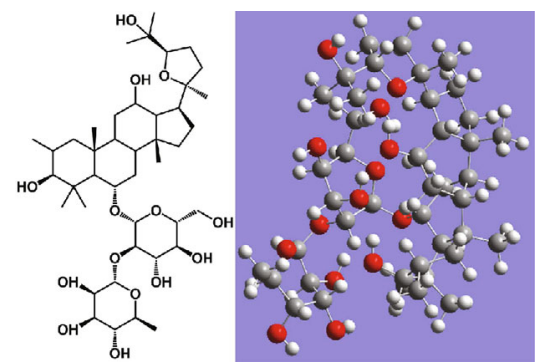

(a)

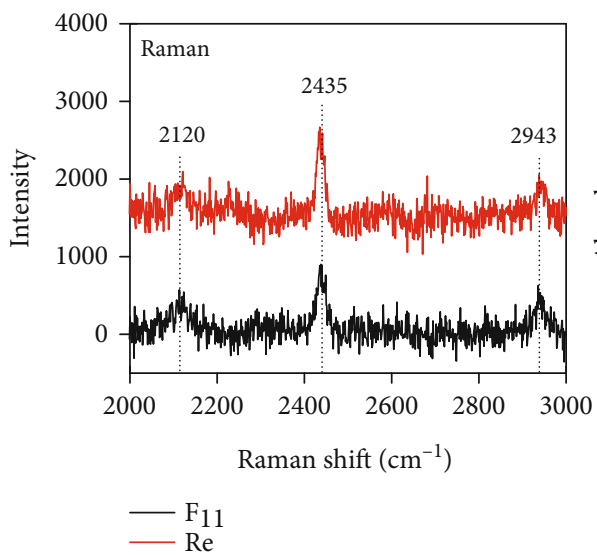

(d)

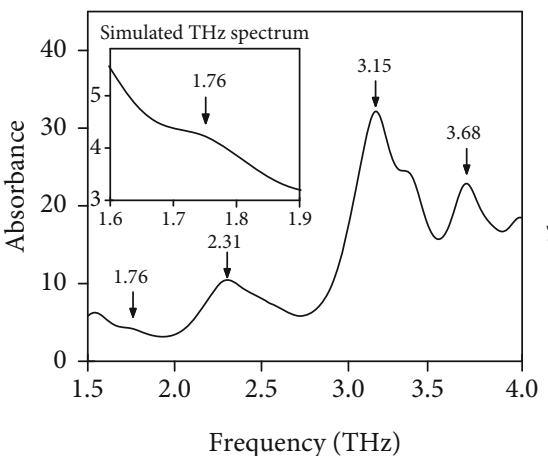

(b)

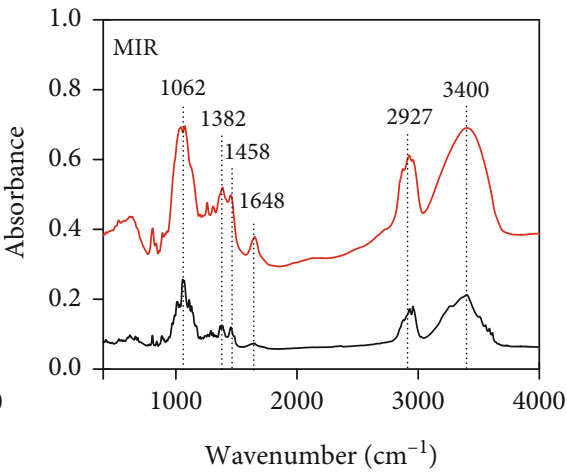

(e)

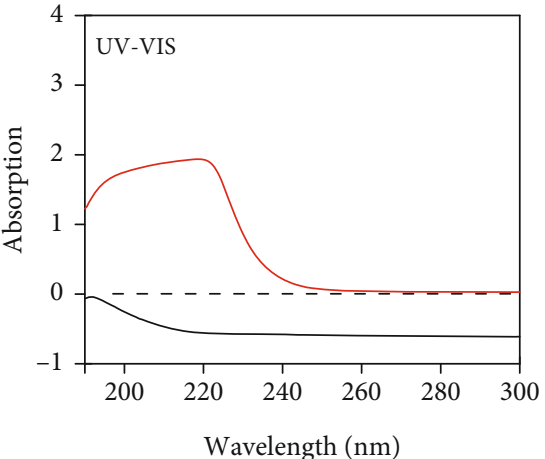

(c)

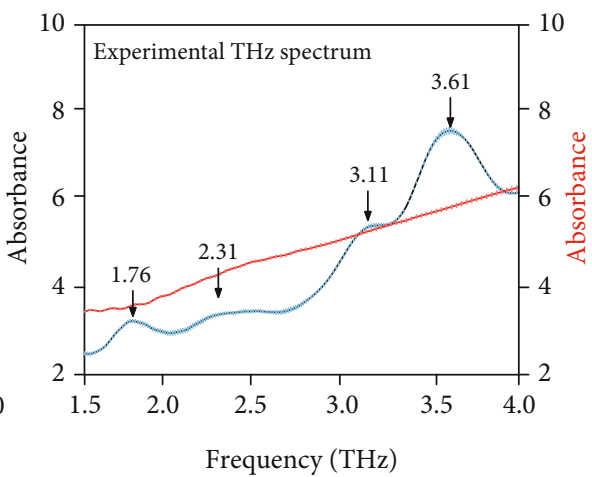

(f)

Figure 1: Different testing methods. Comparison for 24(R)-pseudoginsenoside $\mathrm{F}_{11}$. (a) Molecular formula and theoretical simulation model. (b) Theoretical simulation results. 24(R)-pseudoginsenoside $F_{11}$ and ginsenoside Re (c) UV spectra, (d) Raman spectra, (e) mid-infrared spectra, and (f) terahertz spectra and the corresponding error bars (four tests).

batches from Changbai Mountain, Jilin province, China, to verify the utility of the $\mathrm{F}_{11} \mathrm{THz}$ spectroscopy data for identifying $P$. quinquefolium harvested from different growing regions. For all $P$. quinquefolium samples, the $\mathrm{F}_{11}$ absorption peaks were detected, with the exception of the peak at $2.31 \mathrm{THz}$, which was too small (Figure 2). Moreover, the comparison between the North American and Chinese (Jilin) $P$. quinquefolium samples revealed an extra absorption peak at $2.53 \mathrm{THz}$ for the Chinese batch. This peak may represent trace elements in the soil or reflect other climatic differences between the two examined growing regions [40]. So we can identify Chinese P. Quinquefolium and North American $P$. quinquefolium by using this additional absorption peak at $2.53 \mathrm{THz}$. We also used mid-infrared spectroscopy to do the producing area distinction (Supplementary material 3). However, the mid-infrared spectrum of Panax quinquefolium from different producing areas does not show stable differences in terms of the amplitude and position of absorption peaks. These results prove that the $\mathrm{THz}$ spectroscopy is more suitable and effective for identifying $P$. quinquefolium and for determining the source of the plant material.

3.4. Quantitative Analysis. To confirm the accuracy of our method, we analyzed samples by HPLC-QQQ-MS, which detected the $\mathrm{F}_{11}$ ions in the samples (i.e., ion peaks) (Supple- mentary material 4). This analysis was not influenced by the extent of the conjugation of a structure and was effective for detecting $\mathrm{F}_{11}$.

In this experiment, 14 P. quinquefolium tablets were analyzed to ensure the consistency and comparability of the data. Regarding the $\mathrm{THz}$ spectra, the area of the absorption peaks represents the intensity of the molecular vibration (which is proportional to the concentration). Therefore, establishing the relationship between the peak area and the concentration enables the quantitative analysis of $\mathrm{F}_{11}$ in $P$. quinquefolium. The $\mathrm{F}_{11}$ concentration in P. quinquefolium was determined based on the HPLC-QQQ-MS data. A comparison between the normalized $F_{11}$ concentration data derived from the HPLC-QQQ-MS analysis and the normalized peak areas of the corresponding samples in the $\mathrm{THz}$ spectra (Figure 3(a)) indicated that the relative difference between these two parameters was less than $5 \%$. We subsequently established the relationship between the peak area and the $\mathrm{F}_{11}$ concentration (Figure 3(b)). The results revealed a linear relationship between the peak area and the $\mathrm{F}_{11}$ concentration $\left(R^{2}=0.975\right)$. Therefore, this linear relationship allows for the quantitative analysis of samples based on the peak areas. Because of the chemical loss associated with HPLC-QQQ-MS, we were unable to conduct multiple repetitions of this experiment. Consequently, the error bar and determination coefficient 


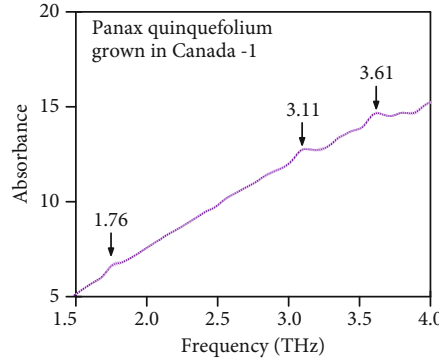

(a)

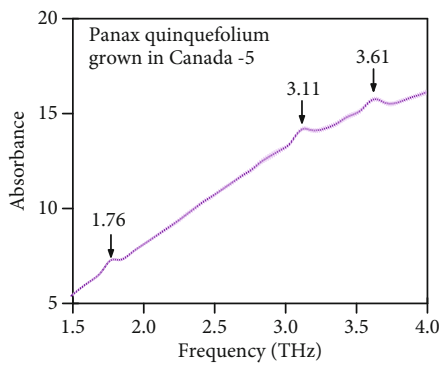

(e)
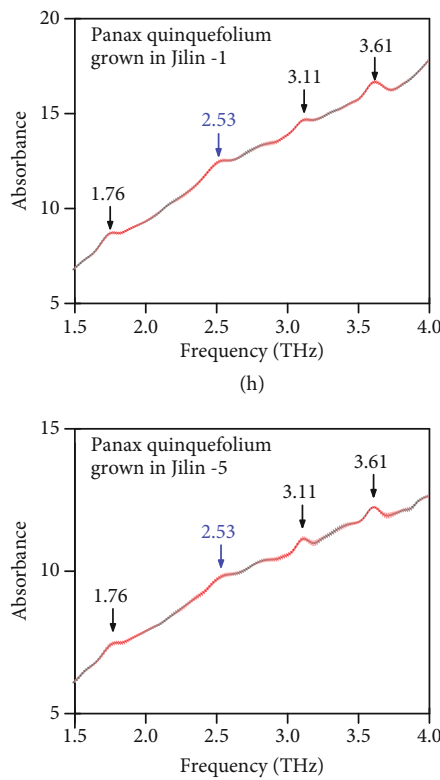

(1)

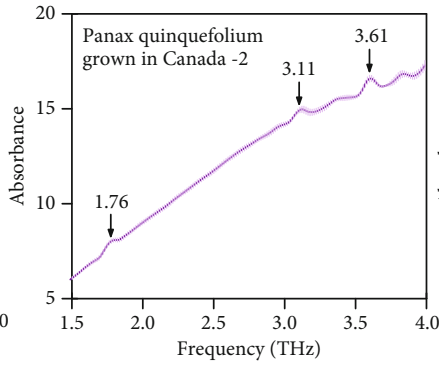

(b)
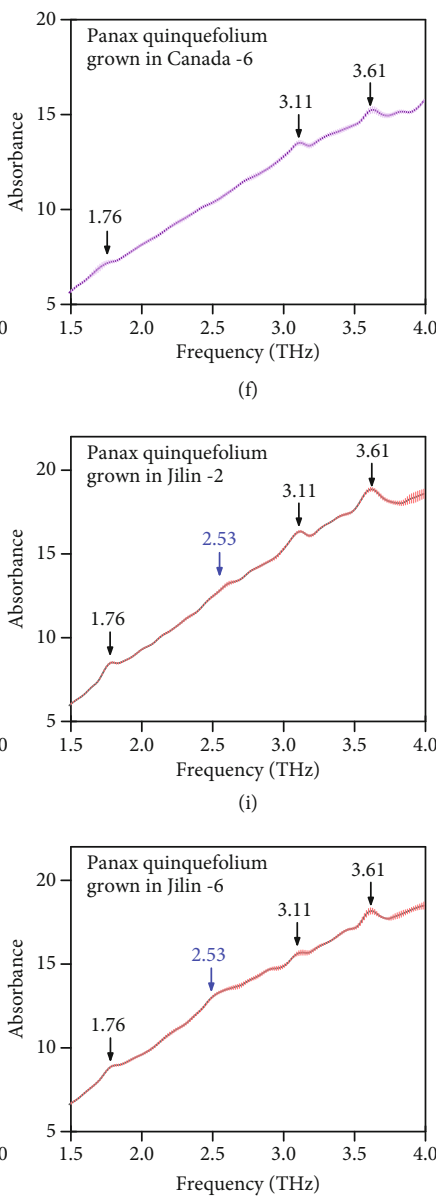

(m)

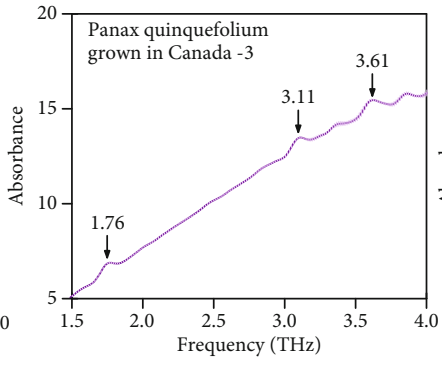

(c)

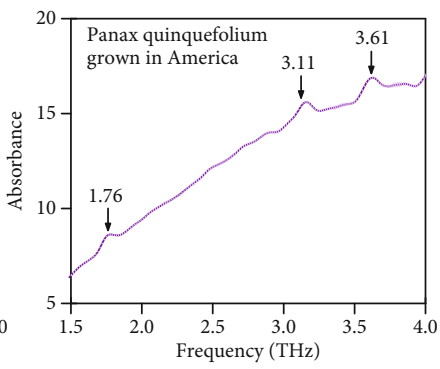

(g)

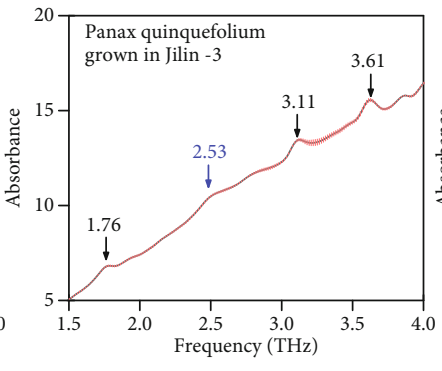

(j)

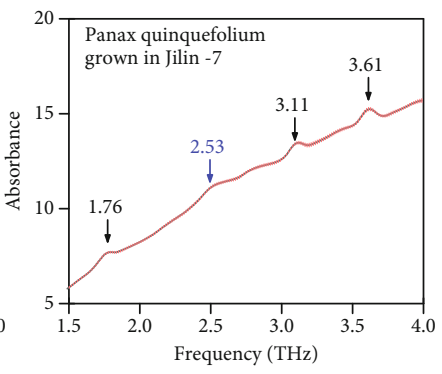

(n)

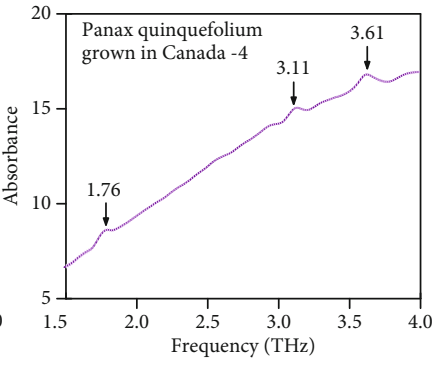

(d)

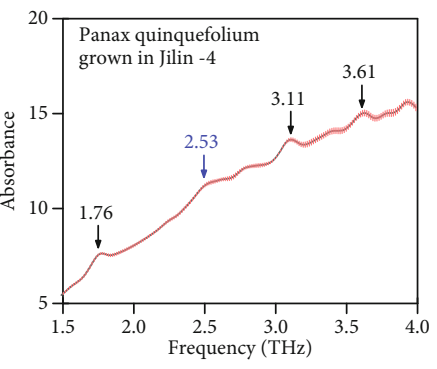

(k)

Figure 2: THz fingerprint spectra. Comparison for Panax quinquefolium from different producing areas. (a-g) THz spectra of North American Panax quinquefolium and the corresponding error bars (four tests). (h-n) THz spectra of Chinese (Jilin) P. quinquefolium and the corresponding error bars (four tests).

could not be determined. These results reflect the potential advantages of our $\mathrm{THz}$ spectroscopic method over other analytical methods (e.g., accurate, rapid, inexpensive, and no chemical losses).

To determine whether the $\mathrm{THz}$ spectrum of P. quinquefolium can be used to identify this species among similar and diverse materials, we performed a PCA to classify and identify samples. The analyzed materials included four batches of ginseng plants produced in 2016 and 2018, four kinds of non-Araliaceae herbs (white peony, red peony, Platycodon grandiflorum, and Atractylodes macrocephala), and 28 other substances (compound paracetamol tablet, morphine hydro- chloride, glutamic acid, lysine, homocysteine, acetaminophen, caffeine, propyphenazone, phenylalanine, benzyl ester, amoxicillin, glimepiride tablet, saccharose, L-tryptophan, glucose, vitamin, aminobutyric acid, tyrosine, creatine, ammonium perchlorate, Intropin, cyclotetramethylenetetranitramine, PETN, trinitrotoluene, RDX (Hexogen), black powder, glucose lozenge, and amino acid lozenge). The corresponding $\mathrm{THz}$ spectra are presented in Supplementary material 5.

Figure 4(a) presents the principal component scores for 50 samples based on the $\mathrm{THz}$ spectral data $\left(\mathrm{PC}_{1}=98.4 \%\right.$ and $\left.\mathrm{PC}_{1}+\mathrm{PC}_{2}=99.4 \%\right)$. When the contribution rate of 


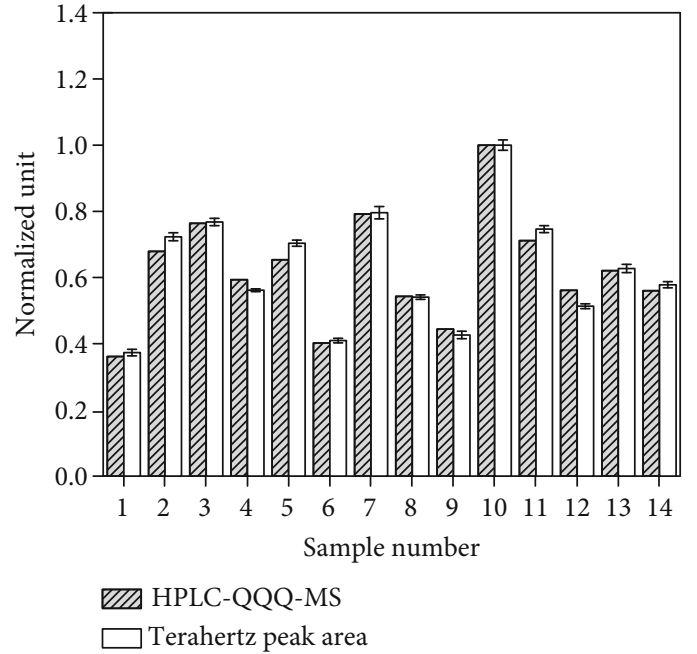

(a)

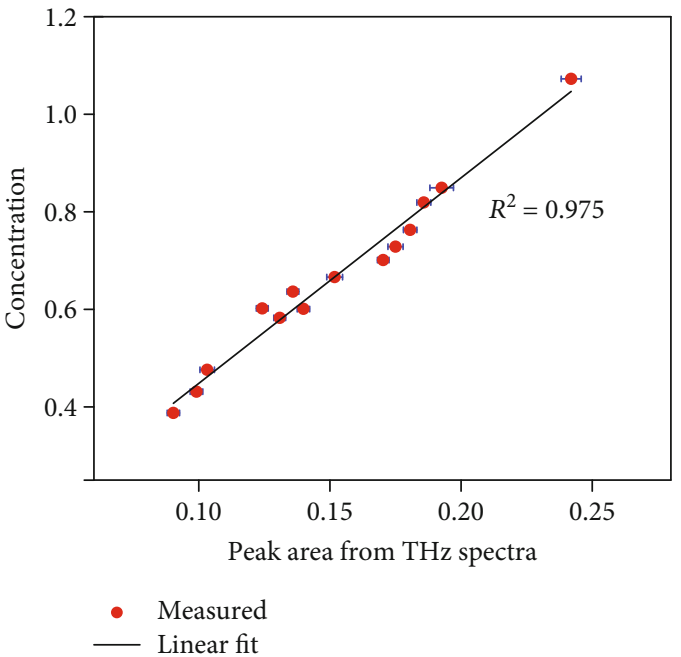

(b)

Figure 3: Quantitative analysis of Panax quinquefolium. (a) Histogram of the comparison between the normalized HPLC and THz spectroscopy data. (b) Analysis of the linear fit between the HPLC and THz spectroscopy data and the corresponding error bars.

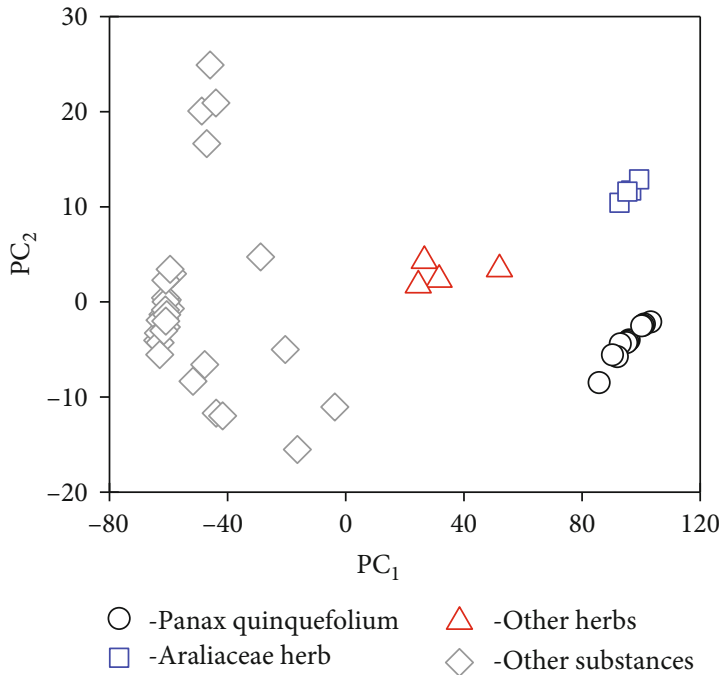

(a)

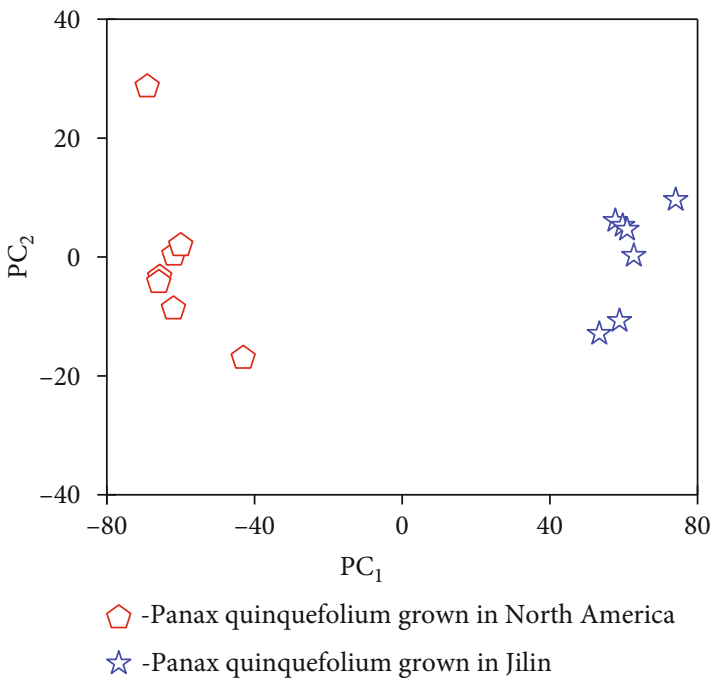

(b)

Figure 4: Principal component analysis based on terahertz spectra. (a) Principal component scores for 50 samples. Black circles, Panax quinquefolium samples; blue squares, ginseng from the family Araliaceae; red triangles, non-Araliaceae herbs; gray rhombi, other substances. (b) Principal component scores for P. quinquefolium from different growing regions. Red pentagons, seven batches of $P$. quinquefolium produced in North America; blue pentacles, seven batches of P. quinquefolium produced in China (Jilin).

the first $p$ principal components is large enough $\left(\mathrm{PC}_{1}+\right.$ $\mathrm{PC}_{2}+\cdots+\mathrm{PC}_{p}>85 \%$ ), the original dataset can be replaced approximately with the first $p$ principal components [36]. Therefore, these spectral data can be analyzed approximately with $\mathrm{PC}_{1}$ and $\mathrm{PC}_{2}$. During the PCA, $\mathrm{PC}_{1}$ represents the largest differences between samples. The greater the $\mathrm{PC}_{1}$ value between two samples, the greater the difference between them [41]. The similarity in the $\mathrm{PC}_{1}$ scores for $P$. quinquefolium and ginseng (Figure $4(\mathrm{a})$ ) is due to similarities in the contents of these Araliaceae herbs (e.g., $\mathrm{Rb}_{1}, \mathrm{Rg}_{1}$, and $\left.\mathrm{Re}\right)$. The observed difference in the $\mathrm{PC}_{2}$ scores is caused by the $\mathrm{F}_{11}$ in $P$. quinquefolium. Additionally, the non-Araliaceae herbaceous plants had $\mathrm{PC}_{1}$ scores that were between those for the Araliaceae herbs and the 28 other substances. The substantial differences in the data for these 28 substances and the data for the Araliaceae herbs and herbaceous plants were explained by the fact that these other substances were derived from completely different species. Figure 4(b) provides the PC scores of $P$. quinquefolium produced in North America and China $\left(\mathrm{PC}_{1}+\mathrm{PC}_{2}=\right.$ 99.9\%). The results indicated that in addition to being used to identify P. quinquefolium, our nonconjugated $\mathrm{F}_{11}$ 
detection method is also useful for distinguishing between $P$. quinquefolium samples collected from diverse growing regions.

\section{Conclusion}

In this study, we developed a new method enabling the accurate, rapid, and cost-effective identification and quantitative analysis of 24(R)-pseudoginsenoside $\mathrm{F}_{11}$. Our theoretical simulation and experimental data proved that $\mathrm{F}_{11}$ can be used as a biomarker for $P$. quinquefolium. Additionally, calculating the peak area can accurately quantify the $\mathrm{F}_{11}$ content in $P$. quinquefolium samples. A comparison between the $\mathrm{THz}$ spectroscopy and HPLC-QQQ-MS data indicated that the results differed by less than $5 \%$, with a determination coefficient of 0.975 . Finally, a PCA revealed that $P$. quinquefolium and other herbs or medicinal plants can be clearly distinguished, with $\mathrm{PC}_{1}$ useful for differentiating between $P$. quinquefolium plants from diverse growing regions.

We herein describe a new method for the qualitative and quantitative analyses of $\mathrm{F}_{11}$ in $P$. quinquefolium to differentiate between $P$. quinquefolium and other herbs or materials. In the future, this method combining $\mathrm{THz}$ energy enhancement or $\mathrm{THz}$ system signal-to-noise ratio improvement [19] and software analysis will pave the way for the identification of various plants, with potential commercial applications.

\section{Conflicts of Interest}

The authors declare no competing financial interest.

\section{Authors' Contributions}

T. K. and Y. P. were major contributors in writing the manuscript. J. Y. and H. L. provide the samples of Panax quinquefolium and the HPLC-QQQ-MS data. J. W., H. C., and L. Z. provide UV and Raman data. Z. W. provides other spectral data for PCA. C. S. and X. W. did the molecular simulation. X. C. provides the XRD test and analysis. Y. P. developed the idea and supervised the project. Y. Z. and S. Z. provide useful advice. All authors revised the paper and approved the final manuscript. Tianyi Kou and Ji Ye contributed equally to this study and share first authorship.

\section{Acknowledgments}

This study was supported by the National Major Project of Scientific Instrument and Equipment Development (2017YFF0106300), the National Natural Science Foundation of China (61922059, 61771314, and 81961138014), the 111 Project (D18014), the International Joint Lab Program supported by Science and Technology Commission of Shanghai Municipality (17590750300), and the Key project supported by Science and Technology Commission of Shanghai Municipality (YDZX20193100004960).

\section{Supplementary Materials}

Supplementary 1 Principal component analysis method. Supplementary 2 Vibration mode analysis. Supplementary 3 Results of Panax quinquefolium MIR test. Supplementary 4 HPLC-QQQ-MS methods and results. Supplementary 5 $\mathrm{THz}$ spectra of substances used in PCA. (Supplementary Materials)

\section{References}

[1] V. Vuksan, J. L. Sievenpiper, V. Y. Y. Koo et al., "American ginseng (Panax quinquefolius l) reduces postprandial glycemia in nondiabetic subjects and subjects with type 2 diabetes mellitus," Archives of Internal Medicine, vol. 160, no. 7, pp. 10091013, 2000.

[2] W. D. Rausch, S. Liu, G. Gille, and K. Radad, "Neuroprotective effects of ginsenosides," Acta Neurobiologiae Experimentalis, vol. 66, no. 4, pp. 369-375, 2006.

[3] J. L. Christensen, "Ginsenosides chemistry, biosynthesis, analysis, and potential health effects," Advances in Food and Nutrition Research, vol. 55, pp. 1-99, 2009.

[4] C. S. Yuan and L. Dey, "Multiple effects of American ginseng in clinical medicine," The American Journal of Chinese Medicine, vol. 29, no. 3, pp. 567-569, 2001.

[5] C.-Z. Wang, S. R. Mehendale, and C.-S. Yuan, "Commonly used antioxidant botanicals: active constituents and their potential role in cardiovascular illness," American Journal of Chinese Medicine, vol. 35, no. 4, pp. 543-558, 2012.

[6] I. Mucalo, D. Rahelić, E. Jovanovski, V. Bozikov, Z. Romic, and V. Vuksan, "Effect of American ginseng (Panax quinquefolius L.) on glycemic control in type 2 diabetes," Collegium Antropologicum, vol. 36, no. 4, pp. 1435-1440, 2012.

[7] J. L. Sievenpiper, J. T. Arnason, E. Vidgen, L. A. Leiter, and V. Vuksan, "A systematic quantitative analysis of the literature of the high variability in ginseng (Panax spp.): should ginseng be trusted in diabetes?," Diabetes Care, vol. 27, no. 3, pp. 839840, 2004.

[8] Y. Jin, A. B. Hofseth, X. Cui et al., "American ginseng suppresses colitis through p53-mediated apoptosis of inflammatory cells," Cancer Prevention Research, vol. 3, no. 3, pp. 339347, 2010.

[9] B. Li, C. Z. Wang, T. C. He, C. S. Yuan, and W. Du, "Antioxidants potentiate American ginseng-induced killing of colorectal cancer cells," Cancer Letters, vol. 289, no. 1, pp. 62-70, 2010.

[10] S. Sun, L. W. Qi, G. J. Du, S. R. Mehendale, C. Z. Wang, and C. S. Yuan, "Red notoginseng: higher ginsenoside content and stronger anticancer potential than Asian and American ginseng," Food Chemistry, vol. 125, no. 4, pp. 1299-1305, 2011.

[11] E. A. Peralta, L. L. Murphy, J. Minnis, S. Louis, and G. L. Dunnington, "American ginseng inhibits induced COX-2 and NFKB activation in breast cancer cells," Journal of Surgical Research, vol. 157, no. 2, pp. 261-267, 2009.

[12] L. W. Qi, C. Z. Wang, and C. S. Yuan, "ginsenosides from American ginseng: chemical and pharmacological diversity," Phytochemistry, vol. 72, no. 8, pp. 689-699, 2011.

[13] X. Wang, T. Sakuma, E. Asafu-Adjaye, and G. K. Shiu, "Determination of ginsenosides in plant extracts fromPanax ginsengandPanax quinquefolius L. by LC/MS/MS," Analytical Chemistry, vol. 71, no. 8, pp. 1579-1584, 1999. 
[14] T. W. D. Chan, P. P. H. But, S. W. Cheng, I. M. Y. Kwok, F. W. Lau, and H. X. Xu, "Differentiation and authentication ofPanax ginseng, Panax quinquefolius, and ginseng products by using HPLC/MS," Analytical Chemistry, vol. 72, no. 6, pp. 1281-1287, 2000.

[15] X. Ma, H. Xiao, and X. Liang, "Identification of ginsenosides in Panax quinquefolium by LC-MS," Chromatographia, vol. 64, no. 1-2, pp. 31-36, 2006

[16] G. Ren and F. Chen, "Simultaneous quantification of ginsenosides in American ginseng (Panax quinquefolium) root powder by visible/near-infrared reflectance spectroscopy," Journal of Agricultural and Food Chemistry, vol. 47, no. 7, pp. 2771-2775, 1999.

[17] C. F. Wu, Y. L. Liu, M. Song et al., "Protective effects of pseudoginsenoside- $\mathrm{F}_{11}$ on methamphetamine-induced neurotoxicity in mice," Pharmacology Biochemistry \& Behavior, vol. 76, no. 1, pp. 103-109, 2003.

[18] X. Yin, B. W. H. Ng, and D. Abbott, Terahertz Imaging for Biomedical Applications, Springer, 2012.

[19] Y. Peng, C. Shi, Y. Zhu, M. Gu, and S. Zhuang, "Terahertz spectroscopy in biomedical field: a review on signal-to-noise ratio improvement," PhotoniX, vol. 1, no. 1, article 12, 2020.

[20] H. Ge, Y. Jiang, F. Lian, Y. Zhang, and S. Xia, "Quantitative determination of aflatoxin B1 concentration in acetonitrile by chemometric methods using terahertz spectroscopy," Food Chemistry, vol. 209, pp. 286-292, 2016.

[21] Z. Wang, J. Wu, W. Yang et al., "Experimental observation of Bethe strings," Nature, vol. 554, no. 7691, pp. 219-223, 2018.

[22] Y. Peng, Y. Zhu, M. Gu, and S. Zhuang, "Terahertz spatial sampling with subwavelength accuracy," Light: Science \& Applications, vol. 8, no. 1, p. 72, 2019.

[23] Y. Peng, C. Shi, M. Xu et al., "Qualitative and quantitative identification of components in mixture by terahertz spectroscopy," IEEE Transactions on Terahertz Science and Technology, vol. 8, no. 6, pp. 696-701, 2018.

[24] X. Wu, Y. Dai, L. Wang et al., "Diagnosis of methylglyoxal in blood by using far-infrared spectroscopy and ophenylenediamine derivation," Biomedical Optics Express, vol. 11, no. 2, pp. 963-970, 2020.

[25] J. El Haddad, F. de Miollis, J. B. Sleiman, L. Canioni, P. Mounaix, and B. Bousquet, "Chemometrics applied to quantitative analysis of ternary mixtures by terahertz spectroscopy," Analytical Chemistry, vol. 86, no. 10, pp. 4927-4933, 2014.

[26] S. Q. du, H. Li, L. Xie et al., "Vibrational frequencies of anti-diabetic drug studied by terahertz time-domain spectroscopy," Applied Physics Letters, vol. 100, no. 14, article 143702, 2012.

[27] P. Szpot, G. Buszewicz, T. Jurek, and G. Teresiński, "Fragmentation patterns involving ammonium adduct fragment ions:a comparison of the determination of metaldehyde in human blood by HPLC-QqQ-MS/MS and UHPLC-Q-TOF-MS," Journal of Chromatography B, vol. 1085, no. 3, pp. 104-109, 2018.

[28] X. Chen, X. Li, K. Pang, X. Fan, Y. Ma, and J. Hu, “Dissipation behavior and residue distribution of fluazaindolizine and its seven metabolites in tomato ecosystem based on SAX SPE procedure using HPLC-QqQ-MS/MS technique," Journal of Hazardous Materials, vol. 342, no. 15, pp. 698-704, 2018.

[29] S. Wold, K. Esbensen, and P. Geladi, "Principal component analysis," Chemometrics and Intelligent Laboratory Systems, vol. 2, no. 1-3, pp. 37-52, 1987.
[30] C. M. Wang, M. Y. Liu, F. Wang et al., “Anti-amnesic effect of pseudoginsenoside- $\mathrm{F}_{11}$ in two mouse models of Alzheimer's disease," Pharmacology Biochemistry \& Behavior, vol. 106, pp. 57-67, 2013.

[31] S. Nakamura, S. Sugimoto, H. Matsuda, and M. Yoshikawa, "Medicinal flowers. XVII. New dammarane-type triterpene glycosides from flower buds of American ginseng, Panax quinquefolium L," Chemical \& Pharmaceutical Bulletin, vol. 55, no. 9, pp. 1342-1348, 2007.

[32] M. Yoshikawa, T. Murakami, K. Yashiro et al., "Bioactive saponins and glycosides. XI. Structures of new dammarane-type triterpene oligoglycosides, quinquenosides I, II, III IV, and V, from American ginseng, the roots of Panax quinquefolium L," Chemical \& Pharmaceutical Bulletin, vol. 46, no. 4, pp. 647-654, 1998.

[33] N. Fuzzati, "Analysis methods of ginsenosides," Journal of Chromatography B, vol. 812, no. 1-2, pp. 119-133, 2004.

[34] A. Wang, C. Z. Wang, J. A. Wu, J. Osinski, and C. S. Yuan, "Determination of major ginsenosides in Panax quinquefolius (American ginseng) using high-performance liquid chromatography," Phytochemical Analysis, vol. 16, no. 4, pp. 272277, 2005.

[35] A. S. Attele, J. A. Wu, and C.-S. Yuan, "Ginseng pharmacology: Multiple constituents and multiple actions," Biochemical Pharmacology, vol. 58, no. 11, pp. 1685-1693, 1999.

[36] M. J. Frisch, G. W. Trucks, H. B. Schlegel et al., Gaussian 09 Revision A.01, Gaussian, Inc., Wallingford, CT, USA, 2019.

[37] M. Baranska, H. Schulz, and L. P. Christensen, "Structural changes of polyacetylenes in American ginseng root can be observed in situ by using Raman spectroscopy," Journal of Agricultural and Food Chemistry, vol. 54, no. 10, pp. 3629$3635,2006$.

[38] T. E. Wallaart, N. Pras, A. C. Beekman, and, and W. J. Quax, "Seasonal variation of artemisinin and its biosynthetic precursors in plants of Artemisia annua of different geographical origin: proof for the existence of chemotypes," Planta Medica, vol. 66, no. 1, pp. 57-62, 2000.

[39] K. Sutthanut, B. Sripanidkulchai, C. Yenjai, and M. Jay, "Simultaneous identification and quantitation of 11 flavonoid constituents in Kaempferia parviflora by gas chromatography," Journal of Chromatography A, vol. 1143, no. 1-2, pp. 227-233, 2007.

[40] H. G. M. Edwards, T. Munshi, and K. Page, "Analytical discrimination between sources of ginseng using Raman spectroscopy," Analytical and Bioanalytical Chemistry, vol. 389, no. 7-8, pp. 2203-2215, 2007.

[41] H. Zhan, S. Wu, R. Bao, L. Ge, and K. Zhao, "Qualitative identification of crude oils from different oil fields using terahertz time-domain spectroscopy," Fuel, vol. 143, pp. 189-193, 2015. 